\title{
Research Article \\ Explicit Conditions for Stability of Nonlinear Scalar Delay Impulsive Difference Equation
}

\author{
Bo Zheng \\ College of Mathematics and Information Sciences, Guangzhou University, Guangzhou 510006, China \\ Correspondence should be addressed to Bo Zheng, zhengbo611@yahoo.com.cn
}

Received 20 March 2010; Accepted 2 June 2010

Academic Editor: Leonid Shaikhet

Copyright (C) 2010 Bo Zheng. This is an open access article distributed under the Creative Commons Attribution License, which permits unrestricted use, distribution, and reproduction in any medium, provided the original work is properly cited.

Sufficient conditions are obtained for the uniform stability and global attractivity of the zero solution of nonlinear scalar delay impulsive difference equation, which extend and improve the known results in the literature. An example is also worked out to verify that the global attractivity condition is a sharp condition.

\section{Introduction and Main Results}

Let $\mathbb{R}, \mathbb{N}$, and $\mathbb{Z}$ be the sets of real numbers, natural numbers, and integers, respectively. For any $a, b \in \mathbb{Z}$, define $\mathbb{Z}(a)=\{a, a+1, \ldots\}$ and $\mathbb{Z}(a, b)=\{a, a+1, \ldots, b\}$ when $a \leq b$.

It is well known that the theory of impulsive differential equations is emerging as an important area of investigation, since it is not only richer than the corresponding theory of differential equations without impulse effects but also represents a more natural framework for mathematical modeling of many world phenomena [1]. Moreover, such equations may exhibit several real-world phenomena, such as rhythmical beating, merging of solutions, and noncontinuity of solutions. And hence ordinary differential equations and delay differential equations with impulses have been considered by many authors, and numerous papers have been published on this class of equations and good results were obtained (see, e.g., [1-10] and references therein).

Since the behavior of discrete systems is sometimes sharply different from the behavior of the corresponding continuous systems and discrete analogs of continuous problems may yield interesting dynamical systems in their own right (see [11-13]), many scholars have investigated difference equations independently. However, there are few concerned with the impulsive difference equations or delay impulsive difference equations (see [14-19]). On the other hand, stability is one of the major problems encountered in applications, but, to the 
best of our knowledge, very little has been done with the stability of impulsive difference equations (see $[15,20])$. Motivated by this, the aim of this paper is devoted to studying the uniform stability and global attractivity of the zero solution of the following nonlinear scalar delay impulsive difference equation:

$$
\begin{gathered}
\Delta x(n)=f\left(n, x_{n}\right), \quad n \in \mathbb{Z}(0), n \neq n_{j}, \\
\Delta x\left(n_{j}\right)=I_{j}\left(x\left(n_{j}\right)\right), \quad j \in \mathbb{Z}(1),
\end{gathered}
$$

where $\Delta$ denotes the forward difference operator defined by $\Delta x(n)=x(n+1)-x(n), f$ : $\left(\mathbb{Z}(0)-\left\{n_{j}\right\}\right) \times S \rightarrow \mathbb{R}, S$ is the set of all functions $\phi: \mathbb{Z}(-k, 0) \rightarrow \mathbb{R}$ for some $k \in \mathbb{N}$, and $x_{n} \in S$ is defined by $x_{n}(m)=x(n+m)$ for $m \in \mathbb{Z}(-k, 0), I_{j}: \mathbb{R} \rightarrow \mathbb{R}$, and $0 \leq n_{1}<n_{2}<\cdots<$ $n_{j}<n_{j+1}<\cdots$, with $n_{j} \rightarrow \infty$ as $j \rightarrow \infty$. By a solution of (1.1), we mean a sequence $\{x(n)\}$ of real numbers which is defined for all $n \in \mathbb{Z}\left(n_{0}-k\right)$ and satisfies (1.1) for $n \in \mathbb{Z}\left(n_{0}\right)$ for some $n_{0} \in \mathbb{Z}(0)$. It is easy to see that, for any given $n_{0} \in \mathbb{Z}(0)$ and a given initial function $\phi \in S$, there is a unique solution of (1.1), denoted by $x\left(n, n_{0}, \phi\right)$ such that

$$
x\left(n_{0}+m, n_{0}, \phi\right)=\phi(m), \quad \text { for } m \in \mathbb{Z}(-k, 0) .
$$

We assume that $f(n, 0) \equiv 0$ and $I_{j}(0) \equiv 0$, so that $x(n) \equiv 0$ is a solution of $(1.1)$, which we call the zero solution.

For $\phi \in S$, define the norm of $\phi$ as

$$
\|\phi\|=\max \{|\phi(j)|: j \in \mathbb{Z}(-k, 0)\},
$$

and for any $H>0$, define

$$
S_{H}=\{\phi \in S:\|\phi\|<H\} .
$$

Definition 1.1. The zero solution of (1.1) is stable, if, for any $\varepsilon>0$ and $n_{0} \in \mathbb{Z}(0)$, there exists a $\delta=\delta\left(n_{0}, \epsilon\right)>0$ such that $\phi \in S_{\delta}$ implies that $\left|x\left(n, n_{0}, \phi\right)\right|<\varepsilon$ for $n \in \mathbb{Z}\left(n_{0}\right)$. If $\delta$ is independent of $n_{0}$, we say that the zero solution of (1.1) is uniformly stable.

Definition 1.2. The zero solution of (1.1) is globally attractive, if every solution of (1.1) tends to zero as $n \rightarrow \infty$.

A simple example of (1.1) is given by

$$
\begin{array}{cl}
\Delta x(n)+C(n) x(n-k)=0, & n \in \mathbb{Z}(0), \quad n \neq n_{j}, \\
\Delta x\left(n_{j}\right)=c_{j} x\left(n_{j}\right), \quad j \in \mathbb{Z}(1),
\end{array}
$$

where $k \in \mathbb{N}, C: \mathbb{Z}(0) \rightarrow \mathbb{R}$, and $\left\{c_{j}\right\}$ is a sequence of real numbers. In [15], the author studied the stability of the zero solution of (1.5), where $C(n) \geq 0$ for $n \in \mathbb{Z}(0)-\left\{n_{j}\right\}$ and $c_{j} \in(-1,0]$, and obtained the following result. 
Theorem 1.3. If

$$
\sum_{i=n-k}^{n} C(i) \prod_{n_{j} \in \mathbb{Z}(i-k, i-1)}\left(1+c_{j}\right)^{-1} \leq \frac{3}{2}, \quad \text { for } n \in \mathbb{Z}(k)
$$

then the zero solution of (1.5) is stable.

In this paper, we assume that there exists a positive constant $H$ and a sequence $\{P(n)\}$ of nonnegative real numbers such that

$$
P(n) M(\phi) \geq-f(n, \phi) \geq-P(n) M(-\phi), \quad \text { for } n \in \mathbb{Z}(0), \phi \in S_{H},
$$

where $M(\phi)=\max \left\{0, \max _{i \in \mathbb{Z}(-k, 0)} \phi(i)\right\}$. Furthermore, we assume that there is a sequence $\left\{b_{j}\right\}$ of positive numbers with $b_{j} \leq 1$ such that

$$
b_{j} x^{2} \leq x\left[x+I_{j}(x)\right] \leq x^{2}, \quad \text { for } j \in \mathbb{Z}(1),|x|<H .
$$

The main purpose of this paper is to establish the following theorems.

Theorem 1.4. Assume that (1.7) and (1.8) hold and

$$
\sum_{i=n-k}^{n} P(i) \prod_{n_{j} \in \mathbb{Z}(i-k, i-1)} b_{j}^{-1} \leq \frac{3}{2}+\frac{1}{2(k+1)}, \quad \text { for } n \in \mathbb{Z}(k) .
$$

Then the zero solution of (1.1) is uniformly stable.

Remark 1.5. Theorem 1.4 generalizes and improves Theorem 1.3 greatly.

The next theorem provides a sufficient condition for every solution of (1.1) tends to zero as $n \rightarrow \infty$, that is, the zero solution of (1.1) is globally attractive.

Theorem 1.6. Assume that (1.7) and (1.8) hold and

$$
\sum_{i=n-k}^{n} P(i) \prod_{n_{j} \in \mathbb{Z}(i-k, i-1)} b_{j}^{-1} \leq \alpha<\frac{3}{2}+\frac{1}{2(k+1)}, \quad \text { for } n \in \mathbb{Z}(k)
$$

and assume that, for each bounded solution $\{x(n)\}$, either

$$
\lim _{n \rightarrow \infty} x(n)>0, \quad \sum_{n=0}^{\infty} f\left(n, x_{n}\right)=-\infty, \quad \text { for } n \in \mathbb{Z}(k),
$$

or

$$
\lim _{n \rightarrow \infty} x(n)<0, \quad \sum_{n=0}^{\infty} f\left(n, x_{n}\right)=\infty, \quad \text { for } n \in \mathbb{Z}(k) .
$$

Then every solution of (1.1) tends to zero as $n \rightarrow \infty$. 
Remark 1.7. An example is worked out in Section 3 to verify that the upper bound $3 / 2+$ $1 / 2(k+1)$ in $(1.10)$ is best possible, that is, the upper bound in (1.10) cannot be improved.

One special form of (1.1) is when

$$
f\left(n, x_{n}\right)=\sum_{j=1}^{m} p_{n, j} x\left(n+k_{n, j}\right)
$$

where $p_{n, j} \leq 0$, and $k_{n, j} \in \mathbb{Z}(-k, 0)$ for $n \in \mathbb{Z}(1), j \in \mathbb{Z}(1, m)$. Then for any $H>0$, (1.7) holds with $P(n)=\sum_{j=1}^{m}\left|p_{n, j}\right|$. As a consequence of Theorem 1.4, we have the following.

Corollary 1.8. Assume that (1.8) holds and

$$
\sum_{i=n-k}^{n} \sum_{j=1}^{m}\left|p_{i, j}\right| \prod_{n_{j} \in \mathbb{Z}(i-k, i-1)} b_{j}^{-1} \leq \frac{3}{2}+\frac{1}{2(k+1)}, \quad \text { for } n \in \mathbb{Z}(k)
$$

Then the zero solution of the equation

$$
\begin{gathered}
\Delta x(n)=\sum_{j=1}^{m} p_{n, j} x\left(n+k_{n, j}\right), \quad n \in \mathbb{Z}(0), n \neq n_{j}, \\
\Delta x\left(n_{j}\right)=I_{j}\left(x\left(n_{j}\right)\right), \quad j \in \mathbb{Z}(1),
\end{gathered}
$$

is uniformly stable.

For the sake of convenience, throughout this paper, we will use the convention

$$
\begin{gathered}
\sum_{n=i}^{j} P(n)=0, \quad \text { whenever } j \leq i-1, \\
\prod_{i \in A} P(i)=1, \quad \text { whenever } A \text { is an empty set, } \\
x(n)=x\left(n, n_{0}, \phi\right) .
\end{gathered}
$$

\section{Proofs of Main Results}

Define

$$
g_{j}(x)= \begin{cases}\frac{x}{x+I_{j}(x)}, & x \neq 0 \\ \frac{1}{b_{j},} & x=0\end{cases}
$$


for $j \in \mathbb{Z}(1)$. Then $1 \leq g_{j}(x) \leq 1 / b_{j}$ for $j \in \mathbb{Z}(1)$ if $|x|<H$. Equation (1.1) can be rewritten as

$$
\begin{gathered}
\Delta x(n)=f\left(n, x_{n}\right), \quad n \in \mathbb{Z}(0), \quad n \neq n_{j}, \\
x\left(n_{j}+1\right)=g_{j}^{-1}\left(x\left(n_{j}\right)\right) x\left(n_{j}\right), \quad j \in \mathbb{Z}(1) .
\end{gathered}
$$

Set

$$
y(n)=x(n) \prod_{n_{j} \in \mathbb{Z}(0, n-1)} g_{j}\left(x\left(n_{j}\right)\right), \quad n \in \mathbb{Z}(-k),
$$

then (2.2) reduces to

$$
\begin{gathered}
\Delta y(n)=f\left(n, x_{n}\right) \prod_{n_{j} \in \mathbb{Z}(0, n-1)} g_{j}\left(x\left(n_{j}\right)\right), \quad n \in \mathbb{Z}(0), n \neq n_{j}, \\
\Delta y\left(n_{j}\right)=0, \quad j \in \mathbb{Z}(1) .
\end{gathered}
$$

And it is easy to see that

$$
|x(n)| \leq|y(n)| \leq|x(n)| \prod_{n_{j} \in \mathbb{Z}(0, n-1)} b_{j}^{-1}, \quad \text { for } j \in \mathbb{Z}(1), n \in \mathbb{Z}(-k)
$$

To prove Theorems 1.4 and 1.6, we need the following lemma.

Lemma 2.1. Let $\{x(n)\}, n \in \mathbb{Z}\left(n_{0}-k\right)$, be a solution of (1.1), $\{y(n)\}$ is defined by (2.3), $n^{*} \in$ $\mathbb{Z}\left(n_{0}+2 k+2\right)$, and $B\left(n^{*}\right)=\max \left\{|y(n)|: n \in \mathbb{Z}\left(n^{*}-3 k-2, n^{*}-1\right)\right\}<H$. If

$$
\sum_{i=n-k}^{n} P(i) \prod_{n_{j} \in \mathbb{Z}(i-k, i-1)} b_{j}^{-1} \leq c+\frac{k+2}{2(k+1)}, \quad \text { for } n \in \mathbb{Z}(k)
$$

holds for some $c \in[(k+2) / 2(k+1), 1]$ and either

$$
y\left(n^{*}\right) \geq 0, \quad y\left(n^{*}\right)>y\left(n^{*}-1\right)
$$

or

$$
y\left(n^{*}\right)<0, \quad y\left(n^{*}\right)<y\left(n^{*}-1\right),
$$

then $\left|y\left(n^{*}\right)\right| \leq c B\left(n^{*}\right)$. 
Proof. We assume that $y\left(n^{*}\right) \geq 0$ and $y\left(n^{*}\right)>y\left(n^{*}-1\right)$. The case where $y\left(n^{*}\right)<0$ and $y\left(n^{*}\right)<$ $y\left(n^{*}-1\right)$ is similar and is omitted.

It is easy to see that Lemma 2.1 holds for $y\left(n^{*}\right)=0$.

If $k=0$, then $c=1$ and $P(n) \leq 2$ for all $n \in \mathbb{Z}(0)$ by (2.6). Thus we only need to prove that $\left|y\left(n^{*}\right)\right| \leq B\left(n^{*}\right)$. Since $y\left(n^{*}\right)>0, y\left(n^{*}\right)>y\left(n^{*}-1\right)$, by (1.7) we have

$$
-P\left(n^{*}-1\right) M\left(-x_{n^{*}-1}\right) \leq-f\left(n^{*}-1, x_{n^{*}-1}\right)<0,
$$

that is,

$$
-P\left(n^{*}-1\right) \max \left\{0,-x\left(n^{*}-1\right)\right\} \leq-f\left(n^{*}-1, x_{n^{*}-1}\right)<0 .
$$

So $x\left(n^{*}-1\right)<0$ which admits that $y\left(n^{*}-1\right)<0$. Hence

$$
\begin{aligned}
y\left(n^{*}\right)-y\left(n^{*}-1\right) & =f\left(n^{*}-1, x_{n^{*}-1}\right) \prod_{n_{j} \in \mathbb{Z}\left(0, n^{*}-2\right)} g_{j}\left(x\left(n_{j}\right)\right) \\
& \leq P\left(n^{*}-1\right) \max \left\{0,-x\left(n^{*}-1\right)\right\} \prod_{n_{j} \in \mathbb{Z}\left(0, n^{*}-2\right)} g_{j}\left(x\left(n_{j}\right)\right) \\
& =-P\left(n^{*}-1\right) y\left(n^{*}-1\right) \leq-2 y\left(n^{*}-1\right),
\end{aligned}
$$

which implies that $y\left(n^{*}\right) \leq-y\left(n^{*}-1\right)$, so $\left|y\left(n^{*}\right)\right| \leq\left|y\left(n^{*}-1\right)\right| \leq B\left(n^{*}\right)$ by the definition of $B\left(n^{*}\right)$.

Now, assume that $y\left(n^{*}\right)>0, y\left(n^{*}\right)>y\left(n^{*}-1\right)$, and $k \geq 1$. By (1.7), we also have

$$
-P\left(n^{*}-1\right) M\left(-x_{n^{*}-1}\right) \leq-f\left(n^{*}-1, x_{n^{*}-1}\right)<0 .
$$

So,

$$
\max \left\{0, \max _{i \in \mathbb{Z}(-k, 0)}-x\left(n^{*}-1+i\right)\right\}>0
$$

Hence, there is $n_{1} \in \mathbb{Z}\left(n^{*}-k, n^{*}\right)$ such that $x\left(n_{1}-1\right)<0$ and $x(n) \geq 0$ for all $n \in \mathbb{Z}\left(n_{1}, n^{*}\right)$. So, $y\left(n_{1}-1\right)<0$ and $y(n) \geq 0$ for all $n \in \mathbb{Z}\left(n_{1}, n^{*}\right)$. For $n \in \mathbb{Z}\left(n_{0}, n^{*}-1\right)$, by (1.7) and (2.4), one has 
Advances in Difference Equations

$$
\begin{aligned}
\Delta y(n) & =f\left(n, x_{n}\right) \prod_{n_{j} \in \mathbb{Z}(0, n-1)} g_{j}\left(x\left(n_{j}\right)\right) \\
& \leq P(n) M\left(-x_{n}\right) \prod_{n_{j} \in \mathbb{Z}(0, n-1)} g_{j}\left(x\left(n_{j}\right)\right) \\
& =P(n) \max \left\{0, \max _{i \in \mathbb{Z}(-k, 0)}\left(-x_{n}(i)\right)\right\} \prod_{n_{j} \in \mathbb{Z}(0, n-1)} g_{j}\left(x\left(n_{j}\right)\right) \\
& =P(n) \max \left\{0, \max _{i \in \mathbb{Z}(-k, 0)}(-x(n+i))\right\} \prod_{n_{j} \in \mathbb{Z}(0, n-1)} g_{j}\left(x\left(n_{j}\right)\right) \\
& =P(n) \max \left\{0, \max _{i \in \mathbb{Z}(n-k, n)}(-x(i)) \prod_{n_{j} \in \mathbb{Z}(0, n-1)} g_{j}\left(x\left(n_{j}\right)\right)\right. \\
& =P(n) \max \left\{0, \max _{i \in \mathbb{Z}(n-k, n)}(-y(i)) \prod_{n_{j} \in \mathbb{Z}(0, i-1)} g_{j}^{-1}\left(x\left(n_{j}\right)\right)\right\} \prod_{n_{j} \in \mathbb{Z}(0, n-1)} g_{j}\left(x\left(n_{j}\right)\right) \\
& \leq B\left(n^{*}\right) P(n) \max _{i \in \mathbb{Z}(n-k, n)} \prod_{n_{j} \in \mathbb{Z}(i, n-1)} b_{j}^{-1} \\
& \leq B\left(n^{*}\right) P(n) \prod_{n_{j} \in \mathbb{Z}(n-k, n-1)} b_{j}^{-1}
\end{aligned}
$$

provided that $\mathbb{Z}\left(n_{0}, n^{*}-1\right)$ contains no impulsive points. And since $\Delta y(n)=0$ if $n$ meets one of impulsive points, we always have

$$
\Delta y(n) \leq B\left(n^{*}\right) P(n) \prod_{n_{j} \in \mathbb{Z}(n-k, n-1)} b_{j}^{-1} \triangleq \bar{P}(n) B\left(n^{*}\right)
$$

for $n \in \mathbb{Z}\left(n_{0}, n^{*}-1\right)$, where $\bar{P}(n)=P(n) \prod_{n_{j} \in \mathbb{Z}(n-k, n-1)} b_{j}^{-1}$.

By the choice of $n_{1}$, there is a real number $\xi \in\left[n_{1}-1, n_{1}\right]$ such that

$$
y\left(n_{1}-1\right)+\left[y\left(n_{1}\right)-y\left(n_{1}-1\right)\right]\left(\xi-n_{1}+1\right)=0 .
$$

Next, we will show that, for any $l \in \mathbb{Z}(0, k)$,

$$
-y(n-l) \leq B\left(n^{*}\right)\left[\sum_{i=n-k}^{n_{1}-1} \bar{P}(i)-\left(n_{1}-\xi\right) \bar{P}\left(n_{1}-1\right)\right], \quad \text { for } n \in \mathbb{Z}\left(n_{1}-1, n^{*}-1\right)
$$


In fact, for any $l \in \mathbb{Z}(0, k)$,

$$
\begin{aligned}
-y(n-l) & =-y\left(n_{1}-1\right)+\sum_{i=n-l}^{n_{1}-2} \Delta y(i)=\Delta y\left(n_{1}-1\right)\left(\xi-n_{1}+1\right)+\sum_{i=n-l}^{n_{1}-2} \Delta y(i) \\
& \leq B\left(n^{*}\right)\left[\left(\xi-n_{1}+1\right) \bar{P}\left(n_{1}-1\right)+\sum_{i=n-l}^{n_{1}-2} \bar{P}(i)\right] \\
& =B\left(n^{*}\right)\left[\sum_{i=n-l}^{n_{1}-1} \bar{P}(i)-\left(n_{1}-\xi\right) \bar{P}\left(n_{1}-1\right)\right] \\
& \leq B\left(n^{*}\right)\left[\sum_{i=n-k}^{n_{1}-1} \bar{P}(i)-\left(n_{1}-\xi\right) \bar{P}\left(n_{1}-1\right)\right]
\end{aligned}
$$

which shows that (2.17) holds.

Substituting (2.17) into (2.4), it is easy to get

$$
\Delta y(n) \leq B\left(n^{*}\right) \bar{P}(n)\left[\sum_{i=n-k}^{n_{1}-1} \bar{P}(i)-\left(n_{1}-\xi\right) \bar{P}\left(n_{1}-1\right)\right], \quad \text { for } n \in \mathbb{Z}\left(n_{1}-1, n^{*}-1\right)
$$

Let

$$
\beta=c+\frac{k+2}{2(k+1)}, \quad d=\sum_{n=n_{1}}^{n^{*}-1} \bar{P}(n)+\left(n_{1}-\xi\right) \bar{P}\left(n_{1}-1\right)
$$

There are two cases to consider.

Case $1(d \leq 1)$. We have by (2.6), (2.16), and (2.19)

$$
\begin{aligned}
y\left(n^{*}\right)= & y\left(n_{1}\right)+\sum_{n=n_{1}}^{n^{*}-1} \Delta y(n)=\left(n_{1}-\xi\right) \Delta y\left(n_{1}-1\right)+\sum_{n=n_{1}}^{n^{*}-1} \Delta y(n) \\
\leq & \left(n_{1}-\xi\right) \bar{P}\left(n_{1}-1\right) B\left(n^{*}\right)\left[\sum_{i=n_{1}-k-1}^{n_{1}-1} \bar{P}(i)-\left(n_{1}-\xi\right) \bar{P}\left(n_{1}-1\right)\right] \\
& +\sum_{n=n_{1}}^{n^{*}-1} \bar{P}(n) B\left(n^{*}\right)\left[\sum_{i=n-k}^{n_{1}-1} \bar{P}(i)-\left(n_{1}-\xi\right) \bar{P}\left(n_{1}-1\right)\right] \\
\leq & B\left(n^{*}\right)\left\{\left(n_{1}-\xi\right) \bar{P}\left(n_{1}-1\right)\left[\beta-\left(n_{1}-\xi\right) \bar{P}\left(n_{1}-1\right)\right]\right. \\
& \left.\left.+\sum_{n=n_{1}} n^{*}-1\right)\left[\beta-\sum_{i=n_{1}}^{n} \bar{P}(i)-\left(n_{1}-\xi\right) \bar{P}\left(n_{1}-1\right)\right]\right\}
\end{aligned}
$$


Advances in Difference Equations

$$
\begin{aligned}
& =B\left(n^{*}\right)\left[\beta d-\sum_{n=n_{1}}^{n^{*}-1} \bar{P}(n) \sum_{i=n_{1}}^{n} \bar{P}(i)-\left(n_{1}-\xi\right) \bar{P}\left(n_{1}-1\right) \sum_{n=n_{1}}^{n^{*}-1} \bar{P}(n)-\left(n_{1}-\xi\right)^{2} \bar{P}^{2}\left(n_{1}-1\right)\right] \\
& =B\left(n^{*}\right)\left[\beta d-\frac{1}{2} d^{2}-\frac{1}{2}\left(\sum_{n=n_{1}}^{n^{*}-1} \bar{P}^{2}(n)+\left(n_{1}-\xi\right)^{2} \bar{P}^{2}\left(n_{1}-1\right)\right)\right] .
\end{aligned}
$$

Since

$$
\begin{aligned}
\sum_{n=n_{1}}^{n^{*}-1} \bar{P}^{2}(n)+\left(n_{1}-\xi\right)^{2} \bar{P}^{2}\left(n_{1}-1\right) & \geq \frac{1}{n^{*}-n_{1}+1}\left[\sum_{n=n_{1}}^{n^{*}-1} \bar{P}(n)+\left(n_{1}-\xi\right) \bar{P}\left(n_{1}-1\right)\right]^{2} \\
& =\frac{1}{n^{*}-n_{1}+1} d^{2} \geq \frac{1}{k+1} d^{2}
\end{aligned}
$$

we have

$$
\begin{aligned}
y\left(n^{*}\right) & \leq B\left(n^{*}\right)\left[\beta d-\frac{1}{2} d^{2}-\frac{1}{2(k+1)} d^{2}\right]=B\left(n^{*}\right)\left[\beta d-\frac{k+2}{2(k+1)} d^{2}\right] \\
& \leq B\left(n^{*}\right)\left(\beta-\frac{k+2}{2(k+1)}\right)=c B\left(n^{*}\right) .
\end{aligned}
$$

Case $2(d>1)$. In this case, there exists $n_{2} \in \mathbb{Z}\left(n_{1}, n^{*}-1\right)$ such that

$$
\sum_{n=n_{2}}^{n^{*}-1} \bar{P}(n) \leq 1, \quad \sum_{n=n_{2}-1}^{n^{*}-1} \bar{P}(n)>1 .
$$

Therefore, we may choose a real number $\eta \in\left(n_{2}-1, n_{2}\right]$ such that

$$
\sum_{n=n_{2}}^{n^{*}-1} \bar{P}(n)+\left(n_{2}-\eta\right) \bar{P}\left(n_{2}-1\right)=1
$$

Notice that

$$
\begin{aligned}
y\left(n^{*}\right)= & {\left[\Delta y\left(n_{1}-1\right)\left(n_{1}-\xi\right)+\sum_{n=n_{1}}^{n_{2}-2} \Delta y(n)+\left(\eta-n_{2}+1\right) \Delta y\left(n_{2}-1\right)\right] } \\
& +\left[\left(n_{2}-\eta\right) \Delta y\left(n_{2}-1\right)+\sum_{n=n_{2}}^{n^{*}-1} \Delta y(n)\right] .
\end{aligned}
$$


So, by (2.6), (2.15), (2.19), and (2.25), we have

$$
\begin{aligned}
y\left(n^{*}\right) \leq & B\left(n^{*}\right)\left[\bar{P}\left(n_{1}-1\right)\left(n_{1}-\xi\right)+\sum_{n=n_{1}}^{n_{2}-2} \bar{P}(n)+\left(\eta-n_{2}+1\right) \bar{P}\left(n_{2}-1\right)\right] \\
& \times\left[\sum_{n=n_{2}}^{n^{*}-1} \bar{P}(n)+\left(n_{2}-\eta\right) \bar{P}\left(n_{2}-1\right)\right]+B\left(n^{*}\right)\left(n_{2}-\eta\right) \bar{P}\left(n_{2}-1\right) \\
& \times\left[\sum_{i=n_{2}-k-1}^{n_{1}-1} \bar{P}(i)-\left(n_{1}-\xi\right) \bar{P}\left(n_{1}-1\right)\right]+\sum_{n=n_{2}}^{n^{*}-1} B\left(n^{*}\right) \bar{P}(n)\left[\sum_{i=n-k}^{n_{1}-1} \bar{P}(i)-\left(n_{1}-\xi\right) \bar{P}\left(n_{1}-1\right)\right] \\
= & B\left(n^{*}\right)\left\{\sum_{n=n_{2}}^{n^{*}-1} \bar{P}(n)\left[\sum_{i=n-k}^{n_{2}-1} \bar{P}(i)-\left(n_{2}-\eta\right) \bar{P}\left(n_{2}-1\right)\right]\right. \\
& \left.+\left(n_{2}-\eta\right) \bar{P}\left(n_{2}-1\right)\left[\sum_{i=n_{2}-k-1}^{n_{2}-1} \bar{P}(i)-\left(n_{2}-\eta\right) \bar{P}\left(n_{2}-1\right)\right]\right\} \\
\leq & B\left(n^{*}\right)\left\{\sum_{n=n_{2}}^{n^{*}-1} \bar{P}(n)\left[\beta-\sum_{i=n_{2}}^{n} \bar{P}(i)-\left(n_{2}-\eta\right) \bar{P}\left(n_{2}-1\right)\right]\right. \\
& \left.+\left(n_{2}-\eta\right) \bar{P}\left(n_{2}-1\right)\left[\beta-\left(n_{2}-\eta\right) \bar{P}\left(n_{2}-1\right)\right]\right\} \\
\leq & B\left(n^{*}\right)\left(\beta-\frac{1}{2}-\frac{1}{2(k+1)}\right)=c B\left(n^{*}\right) .
\end{aligned}
$$

The proof is completed by combining Cases 1 and 2.

Proof of Theorem 1.4. By Lemma 2.1, (1.9) implies that (2.6) holds with $c=1$. For any $\varepsilon \in$ $(0, H)$, assume that $\mathbb{Z}\left(n_{0}, n_{0}+2 k+1\right)$ contains $m=m\left(n_{0}, k\right)$ impulsive points: $n_{0} \leq n_{l+1}<$ $n_{l+2}<\cdots<n_{l+m} \leq n_{0}+2 k+1$. Set

$$
\beta^{\prime}=\frac{3}{2}+\frac{1}{2(k+1)}, \quad \delta=\frac{\varepsilon}{\left(1+\beta^{\prime}\right)^{2 k}}
$$

We will prove that $\phi \in S_{\delta}$ implies that

$$
|x(n)|=\left|x\left(n, n_{0}, \phi\right)\right|<\varepsilon, \quad \text { for } n \in \mathbb{Z}\left(n_{0}\right) .
$$

To this end, we first prove that

$$
|x(n)|<\delta\left(1+\beta^{\prime}\right)^{2 k}(<\varepsilon), \quad \text { for } n \in \mathbb{Z}\left(n_{0}, n_{0}+2 k+1\right) \text {. }
$$


If $n_{l+1}=n_{0}$, then $\left|x\left(n_{l+1}+1\right)\right|=\left|x\left(n_{0}\right)+I_{0}\left(x\left(n_{0}\right)\right)\right| \leq\left|x\left(n_{0}\right)\right|<\delta<\epsilon$. If $n_{l+1}>n_{0}$, then, for $n \in \mathbb{Z}\left(n_{0}, n_{l+1}\right)$, we claim that

$$
|x(n)|<\delta\left(1+\beta^{\prime}\right)^{n_{l+1}-n_{0}}<\epsilon .
$$

In fact,

$$
\begin{aligned}
\left|x\left(n_{0}+1\right)\right| & \leq\left|x\left(n_{0}\right)\right|+\left|f\left(n_{0}, x_{n_{0}}\right)\right|<\delta+P\left(n_{0}\right) \delta \leq \delta\left(1+\beta^{\prime}\right)<\epsilon \\
\left|x\left(n_{0}+2\right)\right| & \leq\left|x\left(n_{0}+1\right)\right|+\left|f\left(n_{0}+1, x_{n_{0}+1}\right)\right| \\
& <\delta\left(1+\beta^{\prime}\right)+\beta^{\prime}\left\|x_{n_{0}+1}\right\| \leq \delta\left(1+\beta^{\prime}\right)+\beta^{\prime} \delta\left(1+\beta^{\prime}\right) \\
& =\delta\left(1+\beta^{\prime}\right)^{2}<\epsilon .
\end{aligned}
$$

In general, we can obtain (2.31) by induction. And so $|x(n)|<\delta\left(1+\beta^{\prime}\right)^{n_{l+1}-n_{0}}(<\epsilon<H)$ for either case with $n \in \mathbb{Z}\left(n_{0}, n_{l+1}\right)$. For $n \in \mathbb{Z}\left(n_{l+1}+1, n_{l+2}\right)$, we have

$$
\begin{aligned}
\left|x\left(n_{l+1}+1\right)\right| & \leq\left|x\left(n_{l+1}\right)\right|+\left|I_{l+1}\left(x\left(n_{l+1}\right)\right)\right| \leq\left|x\left(n_{l+1}\right)\right|<\delta\left(1+\beta^{\prime}\right)^{n_{l+1}-n_{0}}, \\
\left|x\left(n_{l+1}+2\right)\right| & \leq\left|x\left(n_{l+1}+1\right)\right|+\left|f\left(n_{l+1}+1, x_{n_{l+1}+1}\right)\right| \\
& <\delta\left(1+\beta^{\prime}\right)^{n_{l+1}-n_{0}}+\beta^{\prime}\left\|x_{n_{l+1}+1}\right\| \\
& \leq \delta\left(1+\beta^{\prime}\right)^{n_{l+1}-n_{0}}+\beta^{\prime} \delta\left(1+\beta^{\prime}\right)^{n_{l+1}-n_{0}} \\
& =\delta\left(1+\beta^{\prime}\right)^{n_{l+1}-n_{0}+1}<\epsilon .
\end{aligned}
$$

And so

$$
|x(n)|<\delta\left(1+\beta^{\prime}\right)^{n_{l+2}-n_{0}-1}, \quad \text { for } n \in \mathbb{Z}\left(n_{l+1}+1, n_{l+2}\right),
$$

In general, we have

$$
|x(n)|<\delta\left(1+\beta^{\prime}\right)^{n_{l+i+1}-n_{0}-1}, \quad \text { for } n \in \mathbb{Z}\left(n_{l+i}+1, n_{l+i+1}\right), i=1,2, \ldots, m-1 .
$$

Now for $n \in \mathbb{Z}\left(n_{l+m}+1, n_{0}+2 k+1\right)$,

$$
\begin{aligned}
\left|x\left(n_{l+m}+1\right)\right| & \leq\left|x\left(n_{l+m}\right)\right|+\left|I_{l+m}\left(x\left(n_{l+m}\right)\right)\right| \leq\left|x\left(n_{l+m}\right)\right|<\delta\left(1+\beta^{\prime}\right)^{n_{l+m}-n_{0}-1}, \\
\left|x\left(n_{l+m}+2\right)\right| & \leq\left|x\left(n_{l+m}+1\right)\right|+\left|f\left(n_{l+m}+1, x_{n_{l+m}+1}\right)\right| \\
& <\delta\left(1+\beta^{\prime}\right)^{n_{l+m}-n_{0}-1}+\beta^{\prime}\left\|x_{n_{l+m}+1}\right\| \\
& \leq \delta\left(1+\beta^{\prime}\right)^{n_{l+m}-n_{0}-1}+\beta^{\prime} \delta\left(1+\beta^{\prime}\right)^{n_{l+m}-n_{0}-1} \\
& =\delta\left(1+\beta^{\prime}\right)^{n_{l+m}-n_{0}}<\epsilon .
\end{aligned}
$$


And so

$$
|x(n)|<\delta\left(1+\beta^{\prime}\right)^{2 k-1}, \quad \text { for } n \in \mathbb{Z}\left(n_{l+m}+1, n_{0}+2 k+1\right)
$$

which has proved that (2.30) holds. Now, we will prove that

$$
|x(n)|<\epsilon, \quad \text { for } n \in \mathbb{Z}\left(n_{0}+2 k+2\right) \text {. }
$$

Thus we only need to prove that

$$
|y(n)|<\epsilon, \quad \text { for } n \in \mathbb{Z}\left(n_{0}+2 k+2\right) \text {. }
$$

For any $n^{*} \in \mathbb{Z}\left(n_{0}+2 k+2\right)$ and $B\left(n^{*}\right)<H$, we claim that

$$
\left|y\left(n^{*}\right)\right| \leq B\left(n^{*}\right)
$$

In fact, we can assume that $y\left(n^{*}\right) \geq 0$. The case where $y\left(n^{*}\right)<0$ is similar and is omitted. If $y\left(n^{*}\right) \leq y\left(n^{*}-1\right)$, by the definition of $B\left(n^{*}\right),(2.40)$ holds. If $y\left(n^{*}\right)>y\left(n^{*}-1\right)$, then by Lemma 2.1 we have that (2.40) holds.

Since

$$
B\left(n_{0}+2 k+2\right)=\max \left\{|y(n)|: n \in \mathbb{Z}\left(-k, n_{0}+2 k+1\right)\right\}<\varepsilon<H,
$$

by (2.40) we have

$$
\left|y\left(n_{0}+2 k+2\right)\right|<\varepsilon
$$

By repeatedly using (2.40) we get that (2.39) holds. complete.

Combining (2.30) and (2.39), we find that (2.29) holds and the proof of Theorem 1.4 is

Proof of Theorem 1.6. In view of Theorem 1.4, we see that the zero solution of (1.1) is uniformly stable. Therefore, for any $n_{0} \in \mathbb{Z}(0)$, there exists $\delta>0$ such that $\phi \in S_{\delta}$ implies that

$$
|x(n)|=\left|x\left(n, n_{0}, \phi\right)\right|<\frac{1}{2} H, \quad \text { for } n \in \mathbb{Z}\left(n_{0}\right) .
$$

Next, we will prove that

$$
\lim _{n \rightarrow \infty} x(n)=0 .
$$

There are two cases to consider. 
Case 1. $\{x(n)\}$ is eventually positive, that is, there exists $n_{1} \in \mathbb{Z}\left(n_{0}\right)$ such that $x(n)>0$ for all $n \in \mathbb{Z}\left(n_{1}\right)$. Hence, by (1.7) and (1.8), we have $\Delta x(n) \leq 0$ for $n \in \mathbb{Z}\left(n_{1}+k\right)$. That is, $\{x(n)\}$ is eventually nonincreasing and hence $\lim _{n \rightarrow \infty} x(n) \geq 0$. Thus, by (1.11) we see that (2.44) holds. The case when $\{x(n)\}$ is eventually negative is similar and will be omitted.

Case 2. $\{x(n)\}$ is oscillatory in the sense that $\{x(n)\}$ is neither eventually positive nor eventually negative, so $\{y(n)\}$ is also oscillatory. To prove (2.44), we only need to prove that

$$
\lim _{n \rightarrow \infty} y(n)=0
$$

By the proof of Theorem 1.4, we have

$$
|y(n)|<\frac{1}{2} H, \quad \text { for } n \in \mathbb{Z}\left(n_{0}\right) .
$$

Let

$$
\lim _{n \rightarrow \infty} \sup y(n)=p, \quad \lim _{n \rightarrow \infty} \inf y(n)=q,
$$

then $q \leq 0 \leq p$. It suffices to show that

$$
p=q=0
$$

In fact, if (2.48) does not hold, we assume that $p \geq-q$ and $p>0$. The case where $p<-q$ and $q<0$ is similar and is omitted.

Let $0<\varepsilon_{0}<((1-c) / 2) p$, where $c<1$ is given in Lemma 2.1. Then there exists an integer $m_{0} \in \mathbb{Z}(2 k)$ such that

$$
q-\varepsilon_{0} \leq y(n) \leq p+\varepsilon_{0}, \quad \text { for } n \in \mathbb{Z}\left(m_{0}\right)
$$

Since $\lim _{n \rightarrow \infty} \sup y(n)=p>0$ and $\{y(n)\}$ is oscillatory, there must exist an integer $m_{1} \in \mathbb{Z}\left(m_{0}+3 k+2\right)$ such that

$$
y\left(m_{1}\right)>p-\varepsilon_{0}, \quad y\left(m_{1}\right)>y\left(m_{1}-1\right)
$$

By Lemma 2.1 and (2.49) we have

$$
p-\varepsilon_{0}<y\left(m_{1}\right)=\left|y\left(m_{1}\right)\right| \leq c B\left(m_{1}\right) \leq c\left(p+\varepsilon_{0}\right), \quad \text { for } n \in \mathbb{Z}\left(m_{0}\right) .
$$

Equations (2.49) and (2.51) imply that $p-\varepsilon_{0}<c\left(p+\varepsilon_{0}\right)$, which contradicts the fact that $\varepsilon_{0}<((1-c) / 2) p$; thus $(2.48)$ holds and the proof is complete. 


\section{Example}

In this section we will give a result which guarantees that the upper bound $3 / 2+1 / 2(k+1)$ is best possible in Theorem 1.6.

Example 3.1. Consider the delay impulsive difference equation

$$
\begin{gathered}
\Delta x(n)+P(n) x(n-k)=0, \quad n \in \mathbb{Z}(0), n \neq(6 k+1) i, \\
\Delta x\left(n_{i}\right)=b_{i} x\left(n_{i}\right), \quad i \in \mathbb{Z}(0), n_{i}=(6 k+1) i,
\end{gathered}
$$

where $b_{i}=-1 /(i+2)^{2}, i \in \mathbb{Z}(0), k \in \mathbb{Z}(1)$, and $\{P(n)\}$ is a sequence of nonnegative real numbers defined by

$$
P(n)= \begin{cases}0, & n \in \mathbb{Z}((6 k+1) i, k+(6 k+1) i) \\ & \bigcup \mathbb{Z}(3 k+2+(6 k+1) i, 6 k+(6 k+1) i), \\ \frac{1}{k+1}, & n \in \mathbb{Z}(k+1+(6 k+1) i, 2 k+(6 k+1) i) \\ & \bigcup \mathbb{Z}(2 k+2+(6 k+1) i, 3 k+1+(6 k+1) i), \\ c, & n=2 k+1+(6 k+1) i,\end{cases}
$$

where $i \in \mathbb{Z}(0)$ and $c>0$ is an undetermined constant. In view of Theorem 1.6, if

$$
\sum_{i=n-k}^{n} P(i) \prod_{n_{j} \in \mathbb{Z}(i-k, i-1)}\left(1+b_{j}\right)^{-1} \leq c+\frac{k}{k+1}<\frac{3}{2}+\frac{1}{2(k+1)}, \quad \text { for } n \in \mathbb{Z}(k)
$$

or equivalently

$$
c<\frac{k+4}{2(k+1)}
$$

then every solution of (3.1) tends to zero as $n \rightarrow \infty$.

The following theorem shows that if (3.4) does not hold, then there is a solution of (3.1) which does not tend to zero as $n \rightarrow \infty$. This shows that the upper bound $3 / 2+1 / 2(k+1)$ cannot be improved.

Theorem 3.2. Assume that

$$
c \geq \frac{k+4}{2(k+1)}
$$

Then there exists a solution of (3.1) which does not tend to zero as $n \rightarrow \infty$. 
Proof. Let $\{x(n)\}$ be a solution of (3.1) with initial condition of the form

$$
x(n)=\epsilon, \quad \text { for } n \in \mathbb{Z}(-k, 0),
$$

where $\epsilon>0$ is a given constant. Then by (3.1) and the definition of $\{P(n)\}$, we have

$$
\begin{gathered}
x(n)=\left(1+b_{0}\right) \varepsilon, \quad \text { for } n \in \mathbb{Z}(1, k+1), \\
\Delta x(n)=-\frac{1}{k+1}\left(1+b_{0}\right) \epsilon, \quad \text { for } n \in \mathbb{Z}(k+1,2 k) .
\end{gathered}
$$

And hence

$$
\begin{gathered}
x(n)=\left(1+b_{0}\right) \varepsilon\left(2-\frac{n}{k+1}\right), \quad \text { for } n \in \mathbb{Z}(k+1,2 k+1), \\
x(2 k+2)=x(2 k+1)-c x(k+1)=\left(1+b_{0}\right) \varepsilon\left(\frac{1}{k+1}-c\right) .
\end{gathered}
$$

By virtue of (3.1) and (3.8) we get

$$
\Delta x(n)=-\left(1+b_{0}\right) \varepsilon \frac{1}{k+1}\left(2-\frac{n-k}{k+1}\right), \quad \text { for } n \in \mathbb{Z}(2 k+2,3 k+1) .
$$

Summing up from $2 k+2$ to $3 k+1$ and using (3.9), we have

$$
\begin{aligned}
x(3 k+2) & =x(2 k+2)-\frac{\left(1+b_{0}\right) \epsilon}{k+1} \sum_{n=2 k+2}^{3 k+1}\left(2-\frac{n-k}{k+1}\right) \\
& =\left(1+b_{0}\right) \epsilon\left(\frac{1}{k+1}-c\right)-\left(1+b_{0}\right) \epsilon \frac{k}{2(k+1)} \\
& =-\left(1+b_{0}\right) \epsilon\left(c+\frac{k-2}{2(k+1)}\right) \stackrel{\text { def }}{\equiv}\left(1+b_{0}\right) \epsilon_{1} .
\end{aligned}
$$

Furthermore, we have, by the definition of $\{P(n)\}$,

$$
x(n)=\left(1+b_{0}\right) \epsilon_{1}, \quad \text { for } n \in \mathbb{Z}(3 k+2,6 k+1) .
$$

Define the sequence $\left\{\epsilon_{i}\right\}$ as follows:

$$
\epsilon_{0}=\epsilon, \quad \epsilon_{i+1}=-\epsilon_{i}\left(c+\frac{k-2}{2(k+1)}\right), \quad \text { for } i \in \mathbb{Z}(0) .
$$


Then we have by (3.5)

$$
\left|\epsilon_{i+1}\right|=\left|\epsilon_{i}\right|\left|c+\frac{k-2}{2(k+1)}\right| \geq\left|\epsilon_{i}\right|, \quad \text { for } i \in \mathbb{Z}(0)
$$

which implies that $\left\{\left|\epsilon_{i}\right|\right\}$ is a non-decreasing sequence and does not tend to zero as $n \rightarrow \infty$.

Repeating the above argument, we find that, for $n \in \mathbb{Z}(3 k+2+i(6 k+1),(i+1)(6 k+$

$1)), i \in \mathbb{Z}(0)$,

$$
x(n)=\left(1+b_{0}\right)\left(1+b_{1}\right) \cdots\left(1+b_{i}\right) \epsilon_{i+1} .
$$

By the definition of $\left\{b_{i}\right\}$, we know that $\prod_{i=0}^{n}\left(1+b_{i}\right) \nrightarrow 0$ as $n \rightarrow \infty$, so $x(n) \nrightarrow 0$ as $n \rightarrow \infty$. The proof is complete.

\section{Acknowledgments}

The author would like to express her thanks to the referees for helpful suggestions. This research is supported by Guangdong College Yumiao Project (2009).

\section{References}

[1] V. Lakshmikantham, D. D. Bă̌nov, and P. S. Simeonov, Theory of Impulsive Differential Equations, vol. 6 of Series in Modern Applied Mathematics, World Scientific, Teaneck, NJ, USA, 1989.

[2] J. Hale, Theory of Functional Differential Equations, vol. 3 of Applied Mathematical Sciences, Springer, New York, NY, USA, 2nd edition, 1977.

[3] J. J. Nieto and C. C. Tisdell, "On exact controllability of first-order impulsive differential equations," Advances in Difference Equations, vol. 2010, Article ID 136504, 9 pages, 2010.

[4] V. Lakshmikantham, X. Liu, and S. Sathananthan, "Impulsive integro-differential equations and extension of Lyapunov's method," Applicable Analysis, vol. 32, no. 3-4, pp. 203-214, 1989.

[5] Y. Xing, Q. Wang, and D. Chen, "Antiperiodic boundary value problem for second-order impulsive differential equations on time scales," Advances in Difference Equations, vol. 2009, Article ID 567329, 14 pages, 2009.

[6] J. S. Yu, "Stability for nonlinear delay differential equations of unstable type under impulsive perturbations," Applied Mathematics Letters, vol. 14, no. 7, pp. 849-857, 2001.

[7] J. S. Yu, "Explicit conditions for stability of nonlinear scalar delay differential equations with impulses," Nonlinear Analysis: Theory, Methods E Applications, vol. 46, no. 1, pp. 53-67, 2001.

[8] Y. Xing and M. Han, "A new approach to stability of impulsive functional differential equations," Applied Mathematics and Computation, vol. 151, no. 3, pp. 835-847, 2004.

[9] J. Yan, "Stability for impulsive delay differential equations," Nonlinear Analysis: Theory, Methods $\mathcal{E}$ Applications, vol. 63, no. 1, pp. 66-80, 2005.

[10] J. Yan, "Global attractivity for impulsive population dynamics with delay arguments," Nonlinear Analysis: Theory, Methods \& Applications, vol. 71, no. 11, pp. 5417-5426, 2009.

[11] R. M. May, "Simple mathematical models with very complicated dynamics," Nature, vol. 261, no. 5560, pp. 459-467, 1976.

[12] S. Mohamad and K. Gopalsamy, "Dynamics of a class of discrete-time neural networks and their continuous-time counterparts," Mathematics and Computers in Simulation, vol. 53, no. 1-2, pp. 1-39, 2000.

[13] S. Mohamad and K. Gopalsamy, "Exponential stability of continuous-time and discrete-time cellular neural networks with delays," Applied Mathematics and Computation, vol. 135, no. 1, pp. 17-38, 2003.

[14] R. Z. Abdullin, "The comparison method in the stability of nonlinear difference equations with impulse actions," Automation and Remote Control, vol. 61, no. 11, part 1, pp. 1796-1807, 2000. 
[15] Q. Zhang, "On a linear delay difference equation with impulses," Annals of Differential Equations, vol. 18, no. 2, pp. 197-204, 2002.

[16] M. Peng, "Oscillation theorems of second-order nonlinear neutral delay difference equations with impulses," Computers \& Mathematics with Applications, vol. 44, no. 5-6, pp. 741-748, 2002.

[17] Z. He and X. Zhang, "Monotone iterative technique for first order impulsive difference equations with periodic boundary conditions," Applied Mathematics and Computation, vol. 156, no. 3, pp. 605620, 2004.

[18] F. Geng, Y. Xu, and D. Zhu, "Periodic boundary value problems for first-order impulsive dynamic equations on time scales," Nonlinear Analysis: Theory, Methods E Applications, vol. 69, no. 11, pp. 40744087, 2008.

[19] Y. Zhang, J. Sun, and G. Feng, "Impulsive control of discrete systems with time delay," IEEE Transactions on Automatic Control, vol. 54, no. 4, pp. 871-875, 2009.

[20] Q. Q. Zhang and Z. Zhou, "Stability for difference equations of unstable type under impulsive perturbations," Hunan Daxue Xuebao, vol. 29, no. 3, pp. 4-9, 2002 (Chinese). 